\title{
Is the Thiel-embalmed cadaver model a good option for colorectal laparoscopic surgery training programs?A survey on trainees and professors
}

Maria Isabel Prieto-Nieto ( $\square$ iprieto@intermic.com )

Hospital La Paz, Madrid. UAM

Ines Rubio-Perez

Hospital La Paz, Madrid. UAM

Alexander Forero Torres

Hospital La Paz, Madrid. UAM

Beatriz Díaz San Andrés

Hospital La Paz, Madrid. UAM

Luis Asensio Gomez

Hospital La Paz, Madrid. UAM

Hector Guadalajara Labajo

Hospital Fundación Jiménez Diaz. UAM

Francisco Clascá

Universidad Autónoma de Madrid (UAM)

Jaime Ruiz-Tovar

Hospital Fundación Jiménez Diaz. UAM

\section{Research Article}

Keywords: Laparoscopic colorectal surgery, Thiel-embalmed cadavers, Training

Posted Date: July 20th, 2021

DOl: https://doi.org/10.21203/rs.3.rs-701837/v1

License: (1) This work is licensed under a Creative Commons Attribution 4.0 International License.

Read Full License 


\section{Abstract}

Background: Colorectal surgery is one of the most demanding surgical subspecialties, where trainees need to have a deep knowledge and understanding of the anatomy plus acquire specific technical skills. The use of human cadavers as a training modality is a very good option as they are anatomically identical to the patients. The Thiel-embalming method has demonstrated to allow laparoscopic surgical training.

Objective: The aim of this study was to evalutate the idoneity of Thiel embalmed cadavers for training the surgical skills necessary for laparoscopic colorectal surgery.

Design: Prospective observational study

Settings: University Medical Institution

Subjects: Trainees and faculties attending to the training course of laparoscopic colorectal surgery on Thiel-embalmed cadavers

Interventions: A specific questionnaire was designed to evaluate the training experience.

Main outcome measures: 11 questions about the Thiel cadavers, skills, and performance.

Results: 41 participants (24 trainees and 17 teachers) completed the questionnaire. $94.1 \%$ of teachers and $88 \%$ of trainees considered the Thiel cadaver to be a better model for laparoscopic surgery practice than animal models. $82.4 \%$ of the teachers and $72 \%$ of the trainees believed that training with a Thielembalmed cadaver should be a compulsory part of the training before performing colorrectal surgery in vivo. However, $88 \%$ of the trainees and $70.6 \%$ of the teachers considered that this model lacked authenticity due to the absence of bleeding.

Limitations: Data obtained from only one course.

Conclusions: Laparoscopic surgical training using Thiel-embalmed cadaver models can be a realistic and feasible option to develop technical skills in surgical residents and junior consultants.

\section{Introduction}

The arrival of laparoscopic techniques has created a novel paradigm for surgical training, with a new learning model and a shift towards more structured laparoscopic training programs. Trainees need to acquire and perfection new surgical skills before performing in a patient to guarantee the best quality treatment $(1,2)$.

Colorectal surgery is one of the most demanding surgical subspecialties, where trainees need to have a deep knowledge and understanding of the anatomy plus acquire specific technical skills, including the correct use of numerous surgical instruments and devices. Healthcare pressure, increasing costs of 
theater time, and the ethics of learning on human patients have contributed to the demand for teaching programs that allow for the development of basic laparoscopic skills in the laboratory. In the last decade, there has been a development of new synthetic and even virtual training models and their introduction in the market. The efficiency of each type of simulation depends on the complexity of the skills to be taught, the cost and the availability of each simulation model in the teaching center. The use of human cadavers as a training modality is a very good option as they are anatomically identical to the patients. They allow the surgeons to practice safely and to develop all their skills and even go through the full surgical procedures before performing them live (1-3).

The aim of this study was to evalutate the idoneity of Thiel embalmed cadavers for training the surgical skills necessary for laparoscopic colorectal surgery.

\section{Methods}

As part of an advanced colorectal laparoscopic training course that we organized in collaboration with the Department of Anatomy and Neurosciences (School of Medicine, Autonoma University of Madrid, Spain) we planned a prospective observational study to evalute the satisfaction of the training of laparoscopic colorectal approaches on Thiel cadavers.

All methods were carried out in accordance with relevant guidelines and regulations. The human cadavers used in this Project were all obtained from the Body Donation Program of the Autonoma de Madrid University (UAM) http://www.donarcuerpoalaciencia.com. Donors were adults, of both sexes. According to applicable Spanish regulations as well as best practice recommendations from the Spanish Anatomical Society (SAE), everyone had, prior to death, signed a Donation Will for the stated purpose body use for Medical Training and Research. The Donation Will specifically authorized the publication of scientific images taken from the bodies or tissue samples.

All experimental protocols and procedures involving the use of donated bodies for the training activities reported in the present study, were approved by the Ethical committee or institutional review board of Universidad Autónoma de Madrid Body Donation Program Directive Board"

Procedures for the use and disposal of anatomical remains and embalming solutions was supervised by the UAM Health \& Safety Commiitee, following applicable national and local environmental regulations.

Informed consent was obtained from all participants in the training courses for the publication of images taken during the course sessions. A statement to confirm that written informed consent was obtained from all subjects or if subjects are under 18 , from a parent/ legal guardian.

Participants included faculties (expert coloproctologists) and trainees (junior consultants or advanced residents).

\section{Thiel cadaver preparation}


The protocol for Thiel embalming is named after its creator, Professor Walter Thiel, in 1992. It is characterized by a sophisticated process that allows preservation of cadavers, preserving most of the features of a living body. The method consists in a vascular perfusion followed by immersing the body over a period of at least 1-2 months in an embalming fluid containing (in different proportions): boric acid $3 \%$, mono-ethylene glycol $10 \%$, ammonium nitrate $10 \%$, potassium nitrate $5 \%$, stem solution-II $2 \%$, sodium sulfite $7 \%$, and formalin $2 \%$. Differently from fresh-frozed cadavers, Thiel embalmed cadavers last longer, around 3 years, beyond this period they should be cremated. This embalming procedure allows for a better 'life-like' preservation of tissues and textures, like those found in vivo.

\section{Methodology of the Course}

The full-day course included lectures followed by the laboratory training of different laparoscopic colorectal procedures (right hemicolectomy, left hemicolectomy and lower anterior resection of rectum) on the Thiel-embalmed cadavers. Before practicing on the cadaver, the faculty discussed several surgical techniques and details of the anatomy, such as right and left hemicolectomy, anterior rectal resection with total mesorectal excision and abdominoperineal resection. These theoretical sessions were accompanied by instructive videos to improve the understanding of each step.

Surgical procedures were performed on Thiel cadavers in the skills lab of the Autonoma University of Madrid, School of Medicine's Department of Anatomy and Neurosciences. The cadavers were donated for medical education and scientific research throught the University's voluntary body-donation program.

Eight Thiel-embalmed cadavers were prepared for this course and each one was assigned to three trainees resembling a normal colorectal approach, with a surgeon, a first assistant, and a second assistant with the camera (Fig. 1). The cadaver was placed in reverse Trendelenburg position with the legs separated. The laparoscopic devices were provided by sponsors and were equal to those used in a normal hospital operating room (Optics, camera, suction devices, ultrasonic scalpels, endostaplers, sutures, Lone star retractor system, etc.). The three trainees in each group changed their positions during the practice, so that all of them performed a part of the technique as the main surgeon (Fig. 2A). One faculty member was assigned to each group as supervisor to guide the trainees and answer any question arising during the procedures (Fig. 2B).

A specific questionnaire was designed to evaluate the training experience. This questionnaire has been previously validated for the evaluation of laparoscopic surgery courses (4). It evaluated the opinion of both, trainees and faculties and included 11 questions about the Thiel cadavers, skills, and performance. At the end of the course all trainees and Faculties attending the course were requested to answer the questionnaire anonymously.

\section{Results}

The questions included in the questionnaire and the results obtained from both, trainees and faculties are shown in Table 1. There was a total of 41 participants ( 24 trainees and 17 teachers). Among of the 
teachers $35.3 . \%$ were women and $64.7 \%$ were men, whereas among the trainees $56 \%$ were women and $44 \%$ men. Upon evaluation of the participants' training and experience as surgeons, $60 \%$ were residents, $24 \%$ were staff members with $<7$ years experience and $16 \%$ were Staff members with $>7$ years experience. All the faculties were colorectal staff surgeons with over 10 years experience in laparoscopic surgery. All the participants had previous experience with colorectal surgeries on animal models. 
Table 1

Feedback questionnaire (11 questions) and answers provided by course participants, classified as students or Faculty.

Feedback questionnaire $(n=41)$

\section{Question 1}

Had you ever heard of the Thiel embalming method before this course?

\section{Question 2}

If Yes, please chose one of the following:

\section{Question 3}

Can you differentiate the tissues on a Thiel cadaver?

\section{Question 4}

Does the tissue elasticity allow realistic manipulation of the cadaver?

\section{Question 5}

In your opinion, is the appearance of the Thiel cadaver like that of a body in vivo?

\section{Question 6}

Does the lack of bleeding reduce verosimility in the Thiel model?

\section{Question 7}

Is visceral tissue consistency of the Thiel model less than that of the in vivo model?

\section{Question 8}

Do you consider the Thiel cadaver a better model to practice colorectal laparoscopy surgery than pig or other animal models?

\section{Question 9}

Do you believe training with/practicing on a Thiel cadaver should be a compulsory part of the
Answer

Yes

No

I have, but I have never worked with

a Thiel cadaver

I have used a Thiel $\quad 11,1 \% \quad 7,1 \%$ cadaver for anatomic disections

I have used it for laparoscopic training

$36 \%$

$64 \%$

$77,8 \%$

$\begin{array}{ll}\text { Students } & \text { Faculties } \\ (n=24) & (n=17)\end{array}$

$82,4 \%$

$17,6 \%$

$42,9 \%$

Yes

$84 \%$

$94,1 \%$

No

$16 \%$

$5,9 \%$

Yes

$100 \%$

$100 \%$

No

$0 \%$

$0 \%$

Yes

$88 \%$

$94,1 \%$

No

$12 \%$

$5,9 \%$

Yes

$88 \%$

$70,6 \%$

No

$12 \%$

$29,4 \%$

Yes

$44 \%$

$29,4 \%$

No

$56 \%$

$70,6 \%$

Yes

$88 \%$

$94,1 \%$

No

$12 \%$

$5,9 \%$

Yes

$72 \%$

$82,4 \%$ 


\begin{tabular}{lll|}
\hline Answer & $\begin{array}{l}\text { Students } \\
(\mathbf{n = 2 4 )}\end{array}$ & $\begin{array}{l}\text { Faculties } \\
(\mathbf{n}=17)\end{array}$ \\
\hline No & $28 \%$ & $17,6 \%$ \\
\hline Yes & $100 \%$ & $100 \%$ \\
\hline No & $0 \%$ & $0 \%$ \\
\hline Excellent & $80 \%$ & $100 \%$ \\
\hline Good & $20 \%$ & $0 \%$ \\
\hline Average & $0 \%$ & $0 \%$ \\
\hline Poor & $0 \%$ & $0 \%$ \\
\hline Very poor & $0 \%$ & $0 \%$ \\
\hline
\end{tabular}

\section{Question 10}

Do you think the practice with the Thiel cadaver has been useful for the students?

\section{Question 11}

How do you rate the practice with the Thiel cadaver?

When considering the Thiel-embalming method, $82.4 \%$ of the teachers answered that they had heard about it, compared with only $36 \%$ of the trainees. Among the participants who knew this embalming method, $42.9 \%$ of the teachers and $77.8 \%$ of the trainees had never operated on a Thiel-embalmed cadaver: only one teacher $(7.1 \%)$ and one student $(11.1 \%)$ had done previous anatomic disections with cadavers embalmed with this method. When asked if the appearance of the cadaver in this model was like that in vivo, $94.1 \%$ of trainees and $88 \%$ of faculty responded affirmatively.

However, $88 \%$ of the trainees and $70.6 \%$ of the teachers considered that the Thiel model lacked authenticity due to the absence of bleeding and $29.4 \%$ of the teachers felt that visceral tissue consistency was reduced when compared with the human alive.

$94.1 \%$ of teachers and $88 \%$ of trainees considered the Thiel cadaver to be a better model for laparoscopic surgery practice than the pig or other animal models. $82.4 \%$ of the teachers and $72 \%$ of the trainees believed that training with a Thiel-embalmed cadaver should be a compulsory part of the training before performing colorrectal surgery in vivo.

When asked for their opinion about the course, $100 \%$ of the teachers and $80 \%$ of the trainees felt it was excellent and only $20 \%$ of the trainees qualified it as good.

\section{Discussion}

Laparoscopic surgery requires special skills and an increasingly specialized training. Human cadavers have recently begun to be used for laparoscopy training since they provide an anatomic model that resembles a real patient $(1,2)$. Fresh-frozen cadavers have been proposed as a useful model, but they can have increased costs since they must be kept in a large freezer and can present risk of infectious transmission (3). Furthermore, within three hours of de-freezing, the tissue begins to deteriorate, tissue quality decays and, consequently, they can only be used for a limited time. In the search for other 
embalming methods that achieved a better preservation, Thiel developed a formula that importantly reduced smell (when compared to fresh-frozen or formalin) and optimally preserved tissue, color consistency and transparency $(5,6)$. This embalming method is based on water and contains glycol, several salts and conservatives with low levels of formalin ( $0.8 \%$ in the immersion liquid). With the Thiel method, tissue decay is significantly slower, allowing the use of the cadaver for a longer period and repetition of the training sessions if necessary $(7,8)$.

The main reported disadvantage with this embalming method, if compared with animal models, is the lack of blood flow (7). In our course, $88 \%$ of our trainees and $70.6 \%$ of faculty thought that the Thielembalmed cadaver was an unreralistic model due to the lack of bleeding. In addition, $29.4 \%$ of the faculty and $44 \%$ of the trainees felt that the visceral tissue was less consistent/solid than an in vivo model. However, these cons are even more outstanding in any other cadaver models. Animal models can be considered an alternative for cadaveric simulation based on the realistic texture and appearance of the tissues, but the anatomical lay-out of the different structures presents significant differences. The logistics and necessary facilities plus the animal wellbeing policies can weigh against these training models.

Another characteristic of the Thiel method is that it provides exceptional desinfection qualities with a minimal exposure to the noxious chemical products, although the expenses for embalming can be relatively high and the fixation process may take as long as six months. The use of conventional formalin embalming is limited by the resulting tissue rigidity, consistency, and texture, by the strong smell and the impossibility of creating a pneumoperitoneum for laparoscopic training. $(5,8,9)$

Despite the Thiel cadaver model creates good conditions for laparoscopic surgery skill practice and excellent tissue preservation, it is not widely spread, and has only been used in $10 \%$ of the world's Anatomy laboratories $(10,11)$. In the present study only one teacher and one trainee had used a Thiel cadaver for anatomic dissections and only $11.1 \%$ of the trainees had done any practice or laparoscopic procedures with a Thiel cadaver.

Nevertheless, even though Thiel cadavers provide a very appropriate model for surgical training, they have also some limitations that affect their usefulness as a surgical training medium. Firstly, the availability of cadavers is limited by the number of voluntary donations, the high cost of the embalment procedures and the requirement for specific facilities dedicated to cadaver storage, conservation, and dissection. $(2,12,13)$. Secondly, their useful life is restricted, meaning that they must be used within a certain period and only a limited number of surgical procedures can be performed in each cadaver. The quality of cadaver tissue can vary widely depending on the time from death to preservation and the period in storage (1).

Cadaver models with this embalming method have proven to be anatomically superior to pigs in training procedures involving colorrectal surgery $(9,10)$. In our course, $94.1 \%$ of the faculty and $88 \%$ of the trainees considered the Thiel model to be superior to pigs or other animal models for laparoscopic surgery training sessions. Yiasemidou et al described that among surgeons the satisfaction level with 
currently used simulation models was low (65.4\% of the participants in their study were dissatisfied with previous simulation models). However, the participants in their study found Thiel cadavers suitable for surgical simulation, realistic, and have reduced odour. They even concluded that the use of Thiel cadavers can be even more cost-effective that other simulation models ${ }^{14}$. A recently published study conducted on pelvic floor procedures simulation also confirmed our results. The participants recognized that Thiel cadavers present more similarities to patients than other simulation methods. The Thiel cadaveric method was considered by most responders to be the best for the simulation of surgical procedures on the pelvic floor and perineum. Most of the surgeons surveyed recommended conducting these courses with Thiel cadavers for different colleagues in other specialties as a reliable simulation method for training for difficult surgical procedures ${ }^{15}$.

Consequently, whenever possible, cadaveric models should be of choice for postgraduate training in surgical skills, allowing for a better real-life scenario visualization and providing the best opportunity to learn diverse surgical techniques in the human anatomy (3).

Given the high degree of satisfaction of the participating residents and staff in these workshops ( $100 \%$ of the faculty and $80 \%$ of the trainees considered it excellent), a standardized plan for similar courses should be explored to be included in the National Training Programs $(12,14)$. Thiel cadaver courses can be a great tool as a training model to teach, reinforce and improve technical skills in surgical practice. They can also be helpful to evaluate performance of specific procedures and could become part of the skills considered in the end-of training evaluation of surgical residents (8).

Costs associated with the use of a Thiel-embalmed cadaver for medical education are $10-20 \%$ greater than those of a formalin-preserved cadaver, but, as it can be reutilized and preserved without decaying or need for constant refrigeration, the long-term exploitation of a Thiel-embalmed cadaver could potentially be less costly.

Certainly, low incidence or high-risk procedures continue to be a concern in some medical training programs $(9,10)$. Many surgical residents have not been exposed to or performed these uncommon procedures on a patient during their clinical training. This issue has led to the need for alternative training methods that can compensate this lack of clinical exposure and allow the trainees to acquire the necessary skills and practical experience to master different techniques. Possibilities for this include employing simulation or animal and cadaveric models. The Thiel-embalmed cadaver can be a reasonable option to complement the surgical trainee's clinical experience in environments in which not enough of these high-risk or low-frequency procedures are performed. In our course, $82.4 \%$ of the faculty and $72 \%$ of the trainees believed that training with a Thiel cadaver ought to be compulsory during residency training in preparation to performing live colorrectal surgery. This could also allow standarization of surgical procedures and a more precise evaluation of surgical capacities across the surgical training system, something that is essential to guarantee that the surgeons-to-be can perform these procedures safely, precisely, and efficiently $(1,13,14)$. 


\section{Conclusions}

Laparoscopic surgical training using Thiel-embalmed cadaver models can be a realistic and feasible option to help develop technical skills in surgical residents and junior consultants. In colorectal surgery, Thiel-embalmed cadaver models allow mastering of specific operations in human anatomy as a previous step to in vivo performance.

\section{Declarations}

\section{COI/Disclosure:}

The authors have no related conflicts of interest to declare.

\section{Funding/Financial support:}

Medtronic ${ }^{\circledR}$ (USA) provided the energy devices and the staplers for the performance of the surgeries on the cadavers.

\section{Authors"contributions:}

- Data acquisition: Maria Isabel Prieto-Nieto, Ines Rubio-Perez, Alexander Forero Torres, Beatriz Diaz San Ándres Díaz, Luis Asensio Gómez.

- Drafting of manuscript: Maria Isabel Prieto-Nieto, Inés Rubio-Perez, Francisco Clascá, Jaime RuizTovar, Hector Guadalajara Labajo

- Critical revision of manuscript: Francisco Clascá, Jaime Ruiz-Tovar.

\section{References}

1. Usami, T. et al. Assessment of laparoscopic training for gynecological malignancies using Thielembalmed human cadavers. Mol Clin Oncol, 9, 511-514 (2018).

2. Supe, A., Dalvi, A., Prabhu, R. \& Kantharia, C. Bhuiyan P.Cadaver as a model for laparoscopic training. Indian J Gastroenterol, 24, 111-131 (2005).

3. Sharma, M., Macafee, D. \& Horgan, A. F. Basic laparoscopic skills training using fresh frozen cadaver: a randomized controlled trial. Am J Surg, 206, 23-31 (2013).

4. Ruiz-Tovar, J. et al. Training Courses in Laparoscopic Bariatric Surgery on Cadaver Thiel: Results of a Satisfaction Survey on Students and Professors. Obes Surg, 29, 3465-3470 (2019).

5. Thiel, W. The preservation of the whole corpse with natural color. Ann Anat, 174, 185-195 (1992).

6. Thiel, W. Supplement to the conservation of an entire cadaver according to W. Thiel. Ann Anat, 184, 267-269 (2002).

7. McMenamin, P. G. et al. Do We Really Need Cadavers Anymore to Learn Anatomy in Undergraduate Medicine? Med Teach, 40, 1020-1029 (2018). 
8. Sharma, M., Macaffee, D. \& Horgan, A. F. Basic Laparoscopic Skills Training Using Fresh Frozen Cadaver: A Randomized Controlled Trial. Am J Surg, 206, 23-31 (2013).

9. Charlotte, N. C. Cadavers Versus Pigs: Which Are Better for Procedural Training of Surgery Residents Outside the OR? Surgery, 154, 34-37 (2013).

10. Kuroki, T. \& Fujioka, H. Training forl Laparoscopic pancreaticoduodenectomy. Surg Today, 49, 103107 (2019).

11. Kimo Takayesu, J., Peak, D. \& Stearns, D. Cadaver-based training is superior to simulation training for cricothyrotomy and tube thoracostomy. Intern Emerg Med, 12, 99-102 (2017).

12. Bohl, M. A. et al. Evaluation of a Novel Surgical Skills Training Course: Are Cadavers Still the Gold Standard for Surgical Skills Training? World Neurosurg, 127, 63-71 (2019).

13. Miyamoto, R., Oshiro, Y., Nakayama, K. \& Ohkohchi, N. Impact of Three-Dimensional Surgical Simulation on Pancreatic Surgery. Gastrointest Tumors, 4, 84-89 (2018).

14. Yiasemidou, M. et al. Multispecialty Evaluation of Thiel Cadavers for Surgical Training. World J Surg, 41, 1201-1207 (2017).

15. Soler-Silva, A. et al. The Thiel cadaveric model for pelvic floor surgery: Best rated in transferable simulation-based training for postgraduate studies. Eur J Obstet Gynecol Reprod Biol, 256, 165-171 (2021).

\section{Figures}

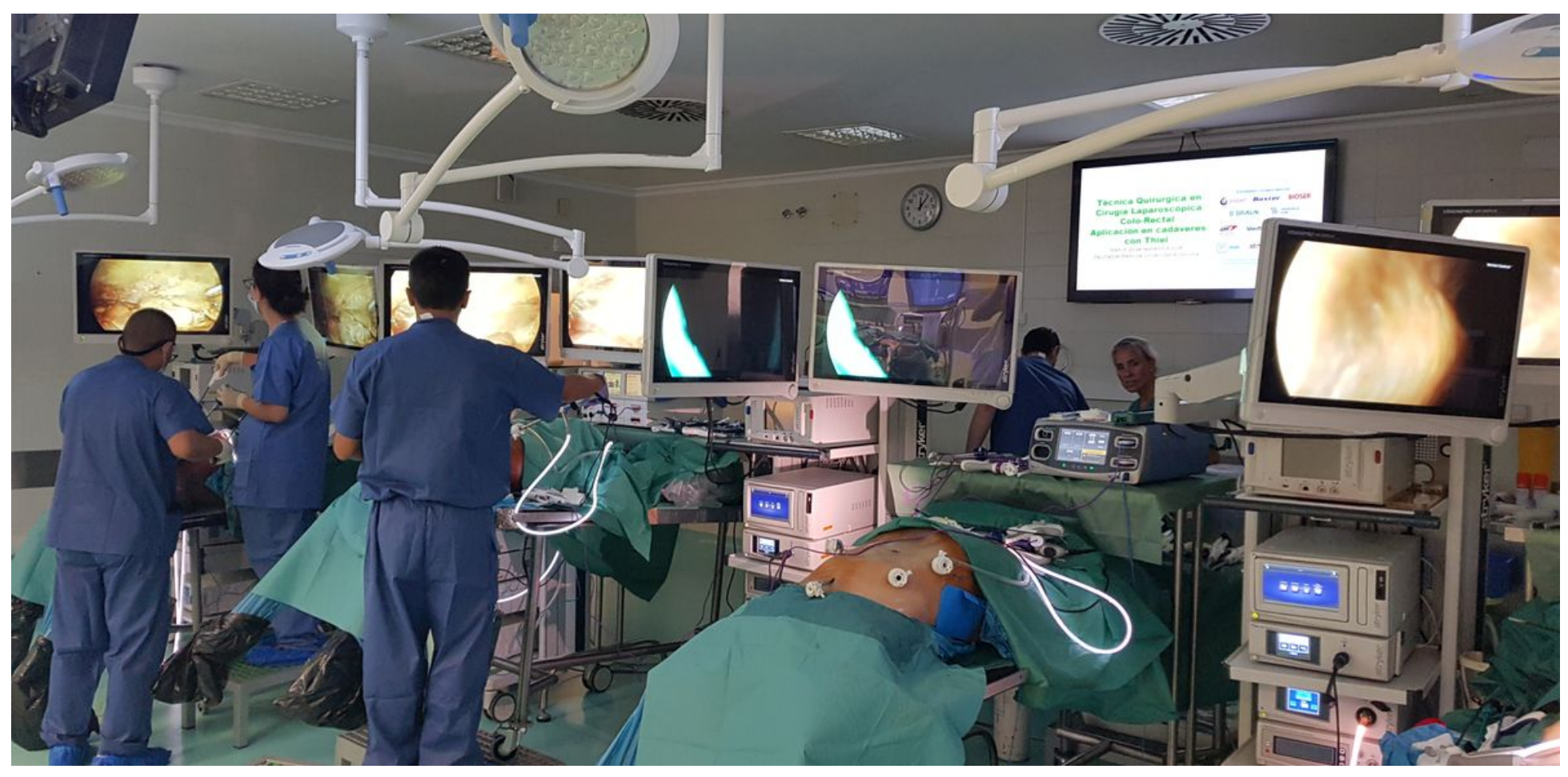

Figure 1 
Set-up of the Thiel cadavers in the cadaver laboratory of the Department of Anatomy and Neurosciences (School of Medicine, Autonoma University of Madrid) for the colorectal surgery skills cours

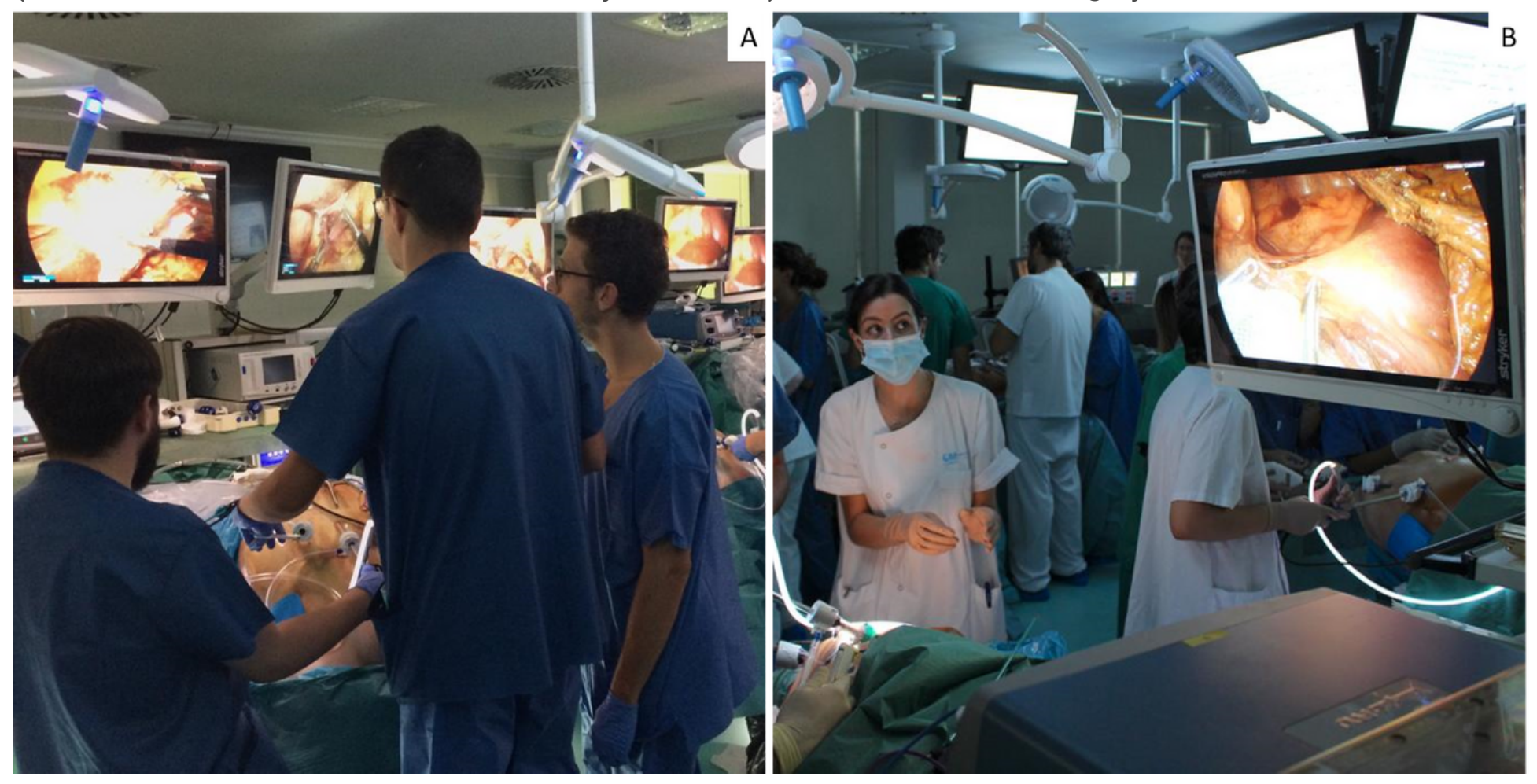

Figure 2

Organization of teams for the course, resembling a normal colorectal approach. Three trainees rotated positions: lead surgeon, first assistant, and a second assistant with the camera. A faculty member guided the steps of each procedure and answered questions. Note the good quality and realistic appearance of the tissues in the screen. 